\title{
Research on Server Cache Mechanism based on P2P Video - on - Demand
}

\author{
LIU Yuling \\ Chongqing College of Electronic Engineering, Chongqing, China,401331
}

Keywords: Server Cache Mechanism, PSP Video, Demand Amount

\begin{abstract}
With the rapid development of computer technology and internet, streaming media technology is widely used in video broadcast, video on demand, distance education and other fields. But the traditional $\mathrm{C} / \mathrm{S}$ architecture service model is often in the case of massive users easily lead to bandwidth bottlenecks and performance bottlenecks. At present, the use of P2P model to solve the streaming media system bottlenecks in the system by more and more researchers concerned. How to improve the quality of service and the scalability of the streaming media system under the condition of making full use of the resources of many peer nodes in the network become a hotspot of P2P streaming media technology.
\end{abstract}

\section{Introduction}

With the growing popularity of computer networks and the rapid development of the user hardware and software upgrades, the rapid development of multimedia technology, the user needs for network resources is not only selected in a single text, voice, images, all dazzling Advertising video, hit the film's wonderful tidbits, blood boiling online game video, friends and other self-timer more and more become an important part of the network. However, the vast majority of media content is used in the traditional way of data content distribution, that is, all users must be from the specified site or server only to download activities, when the complete multimedia file download. As multimedia files tend to occupy a larger storage space, download the entire file takes a long time, the user has to wait too long, unable to meet the user's urgent curiosity. On the other hand, traditional multimedia services can not support real-time programs. The emergence of streaming media technology has effectively changed this situation. The so-called streaming media refers to the use of streaming transmission, in the network in accordance with the order of time and transmission and playback of the continuous time base media formats, such as audio, video and so on. Streaming media compared to the most significant advantage is that users do not have to wait for a long time to all the streaming media files in the local download and then watch, but only part of the data can be downloaded to play.

\section{Streaming Media Technology and P2P Networks}

Streaming media business is divided into two kinds of live and on demand. In the live service, the server only needs to distribute the streaming data to the nodes through IP multicast. And the on-demand service has a high degree of interactivity, the client can select the video to play, and may be drag and drop VCR operation, at the same time the required data is not consistent, it can not apply IP multicast mode, The Even if the use of P2P transmission, IPTV in the on-demand system has been a difficult, slow progress. In 1999, the United Kingdom Video Networks launched the world's first IPTV business; in 2000, the first set of P2P video broadcast system ESM system available; and until 2002 P2Cast transmission protocol proposed, so that the implementation of P2P-based VOD possible. The main factors that affect the performance of P2P VOD system are data cache mechanism and data scheduling algorithm, and the data scheduling algorithm only makes sense after determining the cache mechanism. Therefore, it is necessary to effectively utilize the propagation ability of each node, reduce the load on the server, Improve the performance of the 
entire system, we must study how to more reasonable to the streaming media data cache to the nodes. Existing cache allocation strategies are as follows: based on the popularity of the cache mechanism, based on the probability of the cache mechanism, based on the cache model of the cache mechanism, group-based cache mechanism, and so on. The main problem with these caching strategies is that the caches between the nodes need to be replaced frequently, so that each node must always exchange BM or data cache table, resulting in a relatively large bandwidth waste. For this reason, this paper proposes a cache scrambling scheme based on data scarcity, placing the global cache case on the server by which the data needs to be cached and which data can be replaced from the cache table, and each node only needs to The data request is sent to the server, and the server will indicate that the corresponding node that owns the data sends data to the target node according to the cache condition. Through this scheme, there is no need to exchange BM between the nodes, which greatly reduce the bandwidth occupied; and the cache allocation of each node has a unified scheduling, reducing the redundancy of the data; cache replacement by the server unified scheduling reduced replacement frequency.

\section{Overview of Current Cache Scenarios}

The popularity-based caching model sets the priority by calculating the popularity of each block of video. The popularity is the number of times that time has been downloaded. High popularity of the block is a higher chance of caching, and low popularity of the block is preferred to be replaced. The model is relatively simple, but because the same video within the data block of the popularity of the difference will not be too much, so the download of a single video can not achieve much optimization. In addition, the download frequency for all data blocks to do a global statistics is also a small overhead. Probability-based caching mechanism the main idea is to calculate the relative playtime with the current play point, which data blocks are played at the next highest probability is cached, and the probability is low is replaced. The probability that the data block is cached is determined by the distance from which it is played. The drawback of this scenario is that the frequency of the cache being replaced is too high due to the movement of the play point. Because the cache is often replaced, each node to the neighbor node to download the required data block, you need to exchange the other side of the BM, caused by the bandwidth and delay consumption is correspondingly higher. The gain mechanism based on the gain model sets three gain parameters: media quality gain, start delay gain, and network gain. For each cache, you need to examine the effect of caching the block on the gain of the server, and then prioritize the cache with a larger gain. The disadvantage of this program is that the computational complexity is too high, and the server must master the global cache situation, the computing power for the server is a test. The idea of group-based caching mechanism is to group nodes, each group set up a proxy node, each group of nodes cached data are different, the data request sent by the proxy node, the cache is also calculated by the proxy node data redundancy to manage. The bandwidth and computing capacity of the server under the mechanism is the least, but how to choose the proxy node, and how to ensure the stability of the proxy node is a problem. In addition, the amount of data redundancy is appropriate to require a global consideration and the proxy node only the data of this group of nodes, can not calculate the real appropriate redundancy.

\section{Optimization and Improvement of Cache Management for Streaming Media on Demand System}

P2P streaming media on demand system has the characteristics of high bandwidth and large amount of data transmission. Therefore, how to transfer data to customers is the key problem of streaming media on demand system. In the streaming media on demand system, each user node to contribute their own part of the uplink bandwidth for its maintenance of the neighbors to share their own cache data block. Therefore, the cache management mechanism of the data block has a very important role in improving the overall performance of the system by making better use of the peer node advantage and effectively using the cache data of each user in the system. 
The current streaming media on demand, most of them are the center node of the unstructured P2P architecture. The first arrived node directly requests resources from the source server, and then uses the cache to provide services for the later nodes, and then the node directly to the node earlier than their request resources. User nodes are usually organized into a mesh topology, using distributed management between nodes.

Streaming source server is mainly responsible for caching the entire network of streaming media resources, to the entire coverage network to provide source data, regularly publish new streaming media resources, in the system just started, due to fewer peer nodes, the source server to be responsible for the user The node transmits a large number of data blocks. When the system has a large number of users, each user has buffered some of the resources, this time the data transfer work mainly between the peer nodes, the source server is only responsible for those random node requests. The source server also has the responsibility to regularly update streaming media resources and release current streaming media resources.

Super node is in the ordinary user node to select those who calculate the performance of high, storage capacity, bandwidth stability, online long time, high credibility of the node to play. The super-node may also be one or more servers that are directed by a local streaming media operator. His main role is to help new users join the system to join the system. When a node wants to join the system, the node must first contact the nearest super node in logic. When the super-node examines the node, it divides the node into its own autonomous domain and gives the new node an autonomous domain Neighborhood list. When the node to exit the system, but also to the super node to send out the system message, so that the super node to modify a related record. At the same time, the super node also stores the adjacent super nodes and the streaming media information in the relevant autonomous domain.

Probability cache mechanism does not support the user's VCR operation, but we can see from the previous chapter analysis although the probability cache support for the VCR is not enough, but in order to play a very good playback performance. So here we propose a dynamic partitioning strategy for user cache space.

The first part is used to cache the data blocks before and after the current play position. Its caching strategy uses probability caching. This part of the purpose of the cache is to allow users to operate without the case to ensure smooth video, smooth playback. The cached data block here is called the sequential play-time data block.

The second part of the cache data block is to deal with the user in the operation can also enter the maximum possible to ensure the smooth video, improve video playback continuity. In this part of the cached data is called the data block at the time of operation.

When a node joins the system, it first sends its cache capability and uplink bandwidth to the scheduling server. For a newly added node or a node that is positioned to a data block through a VCR operation, the dispatch server will immediately schedule the node that has the boot play data to send the boot data to the node, and then the node starts video playback and returns the playback time to the scheduled server. The start delay is determined by the time at which the dispatch server finds the corresponding cache node. The cache node of each data block in the dispatch server is stored as an array, so the length of time is $\mathrm{O}$ (1). At every other time period, the dispatch server will calculate the data scarcity once, and then adjust the buffer in the system so that the scatter of each packet is between. The specific practice is to select the partial node from the cache node with the scarcity greater than 1.5 to remove some of its packets from the cache, and then let the cache node in the system buffer the packet with the scarcity of less than 1 until the whole system The scarcity of the full cache or all data is greater than 1 . The dispatch server calculates the required data packets for the next scheduled node in the next time period and distributes the scheduling tasks to the respective cache nodes. The dispatch task dispatches from the TCP channel so that the dispatch server can monitor the abnormal exit status of the cache node. The distribution of scheduling tasks can use the greedy algorithm according to the bandwidth of each node can be distributed in the T period to complete the task. If the upstream bandwidth of the cache node is insufficient, the remaining task is completed by the data server. 


\section{Conclusion}

Broadband computer network technology and the development of streaming media technology, users of the use of the network and information needs, not only stay in the information query and static text content, began to appreciate the audio-visual appreciation, making video on demand increasingly popular. Despite the continuous expansion of network bandwidth, video server I/O bandwidth, processing capacity is also rising, but the bandwidth resources in the hardware construction is limited, can not meet the growing demand for transmission media data, Users often cause server I/O bandwidth or network bandwidth bottlenecks. In order to break through the bandwidth bottleneck, this paper studies how to effectively through the P2P network, the use of effective cache strategy design, so that limited resources for as many users to provide services.

\section{References}

[1] Li Weizhang. P2P technology and application[J]. Telecommunications Technology, 2006 (04)

[2] Sun Pengfei, ZHANG Chengwen, TAN Xuezhi .Application of IP Multicast in Softswitch of Cluster[J]. Electronic Devices, 2005 (02)

[3] An Hui, Chen Xinmeng, Jia Ying. Research and Implementation of Streaming Media Agent Cache [J]. Computer Engineering, 2005 (10)

[4] Gong Haigang, Liu Ming, Xie Li. Review of the progress of P2P streaming media transmission [J] .Computer Science. 2004 (09)

[5] Zhang Lianfeng, Liu Naian, Qian Xiu Penang, Zhang Yuqing. Review: peer-to-peer (P2P) technology [J]. Computer Engineering and Applications, 2003 (12)

[6] Zhao Zhongbai, Jiang Xiu, Wu Zhimei. IP Multicast Technology and Its Application in VoD System[J]. Computer Engineering, 2002 (07) 